\title{
2D PCA-based Localization for Mobile Robots in Unstructured Environments
}

\author{
F. Carreira, C. Christo, D. Valério, M. Ramalho, C. Cardeira, J. M. F. Calado, and P. Oliveira
}

\begin{abstract}
In this paper a new PCA-based positioning sensor and localization system for mobile robots to operate in unstructured environments (e.g. industry, services, domestic...) is proposed and experimentally validated. The inexpensive positioning system resorts to principal component analysis (PCA) of images acquired by a video camera installed onboard, looking upwards to the ceiling. This solution has the advantage of avoiding the need of selecting and extracting features. The principal components of the acquired images are compared with previously registered images, stored in a reduced onboard image database, and the position measured is fused with odometry data. The optimal estimates of position and slippage are provided by Kalman filters, with global stable error dynamics. The experimental validation reported in this work focuses on the results of a set of experiments carried out in a real environment, where the robot travels along a lawn-mower trajectory. A small position error estimate with bounded co-variance was always observed, for arbitrarily long experiments, and slippage was estimated accurately in real time.
\end{abstract}

\section{INTRODUCTION}

The problem of localization has been a great challenge to the scientific community in the area of mobile robotics; see [4], [1] and the references therein.

This paper follows an approach resorting to Principal Component Analysis (PCA) that actually does not depends on any predefined structure of the environment. Of course, there should always be something to distinguish data acquired in one location to data acquired in another location but no previous assumptions on the predefined structure of the environment needs to be considered. The PCA data analysis corresponds to the computation of the data orthogonal components that will make each dataset different. Hence, the localization is defined based on the PCA of the large amount of data taken from the unstructured environment.

The use of vision systems for robot localization is very common due to the ability to obtain information about the environment. Many vision systems compute the robot pose (position and attitude) from features of the environment. Since such feature based techniques are computationally heavy, the use of PCA in mobile robots for self-localization

C. Christo, D. Valério, M. Ramalho and C. Cardeira are with IDMEC / Instituto Superior Técnico, Technical University of Lisbon, Av. Rovisco Pais 1, 1049-001 Lisboa Portugal, cchristo@dem.ist.utl.pt, \{duarte.valerio, mramalho, carlos. cardeira\}eist. utl.pt

F. Carreira and J. Calado are with IDMEC / Instituto Superior Técnico, Technical University of Lisbon and with Instituto Superior de Engenharia de Lisboa / IPL, R. Conselheiro Emídio Navarro 1, 1959-007 Lisboa, \{fcarreira, jcalado\}@dem. isel.ipl.pt

P. Oliveira is with ISR / Instituto Superior Técnico and IDMEC / Instituto Superior Técnico, Technical University of Lisbon, address as above, p.oliveiraddem.ist.utl.pt has been explored, but using front or omnidirectional cameras, causing the algorithms to address problems of occlusion or comparison with images in different planes. In [5], PCA was used for terrain reference navigation of underwater vehicles. The PCA-based localization system that we present is this work corresponds to a experimental validation of the one proposed in [5], using a Dubins Car equipped with a video camera looking upwards to the ceiling.

Beyond the problems of image processing for selflocalization, another challenge is to deal with the fusion of the PCA-based position with the odometry data that is given by the robot kinematics. Mobile robot kinematics (e.g. Dubins car) are in general non linear. This fact prevents the direct use of a Kalman Filter, which is a linear optimal estimator. To tackle this problem, many localization systems use the Extended Kalman Filter (EKF) with well characterized optimality and stability limitations. Even though it can give a reasonable performance, the EKF may diverge in consequence of wrong linearization or sensor noise.

In this paper, we use a Linear Parameter Varying (LPV) model for the Differential Drive Car, thus avoiding the non-linear model issues mentioned above. Moreover, the filter also estimates the slippage that is eventually present in the reality. Many researchers tend to neglect slippage: our approach addresses the problem explicitly. As slippage is inevitable, we append a state to our model to express the slippage explicitly. The linear time-varying Kalman filter adopted estimates both slippage and robot localization. Furthermore, the optimal estimate is achieved, under the assumption that disturbance noise can be modeled by Gaussian distributions, with global stable error dynamics can be obtained [5].

The proposed PCA-based position sensor and localization estimation is a generalization of the 1D system presented in [3]; for a more detailed description see technical report [2]. It has the following advantages:

- The robot is able to self-locate in an indoor environment, only with onboard sensors (no external sensors or landmarks are required).

- The algorithm is fast, thus it consumes very few computational resources.

- The database of images stored onboard the mobile robot is of reduced size, when compared with the total number of images considered ( $89 \%$ reduction in our case).

- The memory to allocate for the database storage is flexible and related with the required positioning error accuracy.

- No hypothesis is made about specific features in the 


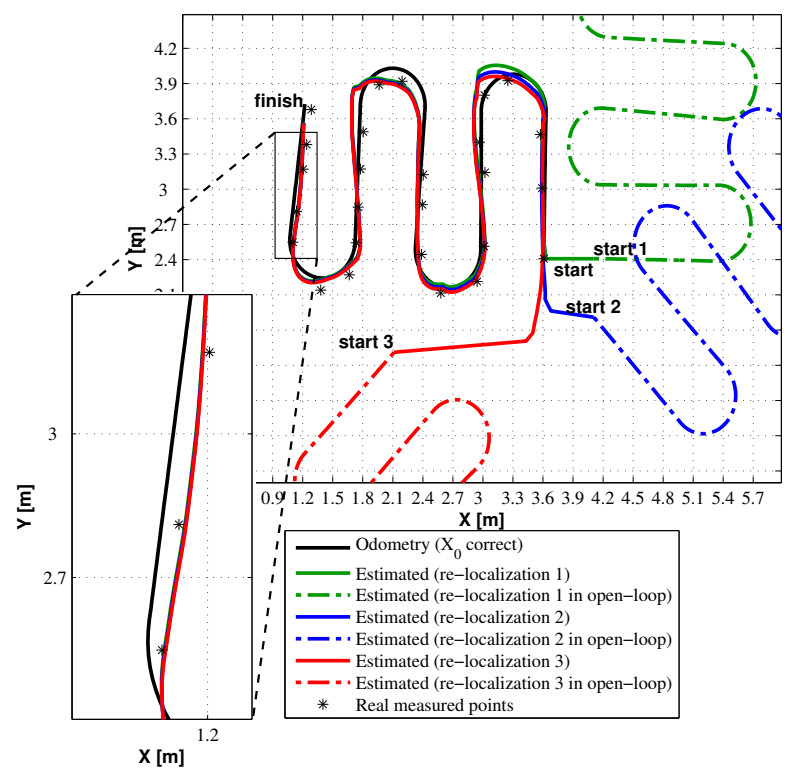

Fig. 1. Results of stability tests considering wrong initial position and attitude

environment: thus this system can operate in an unstructured environment where the only requirement is that images must be different in each location.

- Under Gaussian assumption for the disturbances, the localization system estimates in real time the position and slippage with global stable error dynamics.

Limitations for the proposed approach include:

- The robots should work in buildings with static ceilings where rich information can be found (e.g. HVAC, electrical and security systems, etc.); thus, in general, it cannot be used outdoors.

- The system is formulated in a digital discretized version as well as the PCA approach pursued.

- A general limitation of all vision-based systems is their sensitivity relative to lighting conditions. However, a mix of image and distance (e.g. using time of flight or structured light cameras) would increase the robustness to lighting conditions.

\section{EXPERIMENTAL RESULTS}

To test the estimator a lawn-mower trajectory was employed with a $0.1 \mathrm{~m} \cdot \mathrm{s}^{-1}$ robot speed, a $5 \mathrm{~Hz}$ sample frequency, and the Kalman filter estimation initialized with a wrong robot position $X_{0}$ and a wrong robot attitude. Figure 1 shows that the estimator gives a very good approximation of the real trajectory: results remain stable and convergence with the real trajectory is very fast. The inset shows that the real robot position is inside the estimated uncertainty ellipsoids around points of the estimated trajectory. Figure 2 shows the same happens with an imposed angular slippage of $1 \% \mathrm{~s}$; this slippage is estimated correctly after $40 \mathrm{~s}$.

A high definition version of the video submitted to this conference, showing the performance of the localization system, can be downloaded at http://www.dem.ist. utl.pt/ cardeira/Mesh/Videos/Videos.htm.
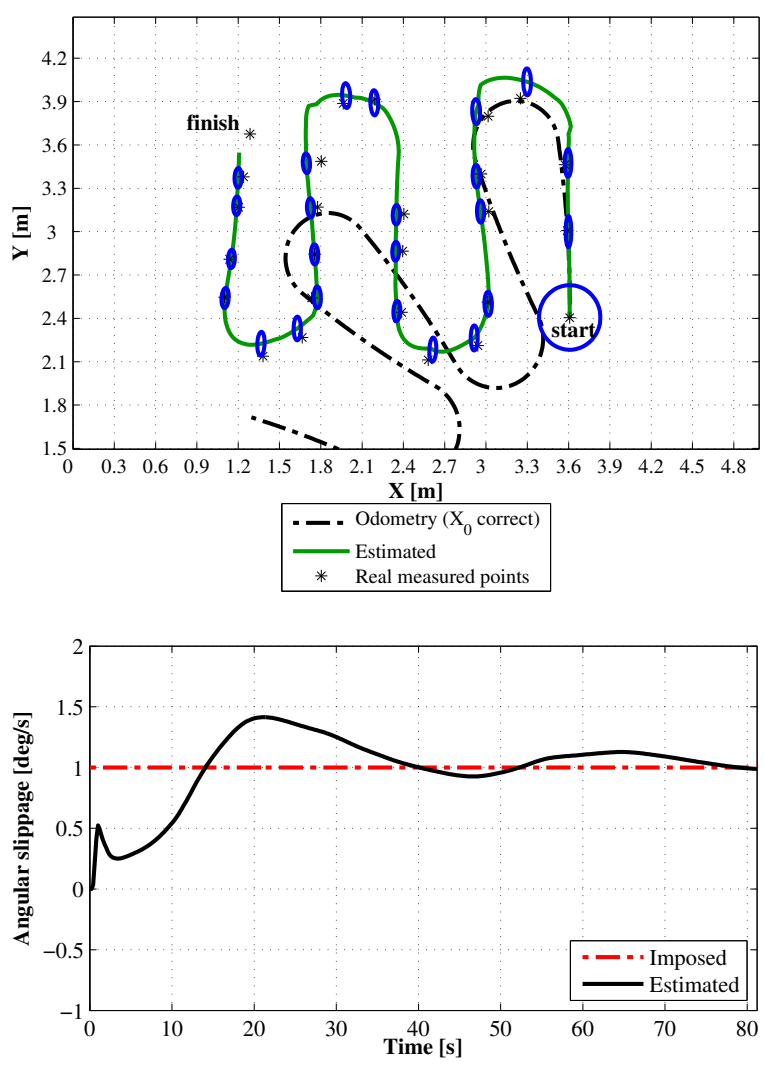

Fig. 2. Results of position (top) and angular slippage (bottom) estimation; the angular slippage is imposed

\section{FUTURE WORK}

This paper represents the initial step towards an agent based architecture where a large set of mobile robots will be able to cooperate to perform navigation and formation tasks, featuring obstacle avoidance and human interaction in different environments.

\section{ACKNOWLEDGMENT}

This work was supported by the Strategic Project PEstOE/EME/LA0022/2011, through FCT (Unit IDMEC - IST, Research Group CSI/IDMEC/LAETA).

\section{REFERENCES}

[1] T. Bailey and H. Durrant-Whyte. Simultaneous localization and mapping (SLAM): part II. IEEE Robotics Automation Magazine, 13(3):108117, 2006.

[2] F. Carreira, C. Christo, D. Valério, M. Ramalho, C. Cardeira, J. M. F. Calado, and P. Oliveira. Experimental validation of a PCA-based localization system for mobile robots in unstructured environments. IDMEC/CSI Internal Report, http://www.dem.ist.utl.pt/ cardeira/Mesh/Ca12b.pdf.

[3] F. Carreira, C. Christo, D. Valério, M. Ramalho, C. Cardeira, J. M. F. Calado, and P. Oliveira. Experimental validation of a PCA-based localization system for mobile robots in unstructured environments. In Robotica-12th International Conference on Autonomous Robot Systems and Competitions, Guimarães, 2012.

[4] H. Durrant-Whyte and T. Bailey. Simultaneous localization and mapping: part I. IEEE Robotics Automation Magazine, 13(2):99-110, 2006.

[5] Paulo Oliveira. MMAE terrain reference navigation for underwater vehicles using PCA. International Journal of Control, 80(7):10081017, 2007. 